\title{
TOPOLOGICAL ASPECTS OF ORDER IN $C(X)$
}

\author{
M. KANDIĆ AND A. VAVPETIČ
}

\begin{abstract}
In this paper we consider the relationship between order and topology in the vector lattice $C(X)$ of all continuous functions on a Hausdorff space $X$. We prove that the restriction of $f \in C(X)$ to a closed set $A$ in the case when $X \in T_{3 \frac{1}{2}}$ induces an order continuous operator iff $A=\overline{\operatorname{Int} A}$. This result enables us to easily characterize bands and projection bands in $C_{0}(X), C_{b}(X)$ and $C(X)$. Our results serve us to provide a positive answer to the question on lifting un-convergence from closed ideals of $C_{0}(X)$ and $C_{b}(X)$.
\end{abstract}

\section{INTRODUCTION}

The interactions between order and topology on spaces of continuous functions have been studied extensively in the past. Although closed ideals, bands and projection bands in $C(X)$ where $X$ is compact and Hausdorff have nice characterizations through closed sets of $X$, the lattice $C(X)$ is very rarely order complete. Order completeness of the lattice $C(X)$ is equivalent to the fact that $X$ is extremally disconnected MN91.

In Section 3 we consider the operator $\Phi_{A}: C(X) \rightarrow C(A)$ which maps a continuous function on $X$ to its restriction on $A \subseteq X$. Although this operator is a contraction between $C_{b}(X)$ and $C_{b}(A)$, in general it is not order continuous. We prove that, whenever $X \in T_{3 \frac{1}{2}}$ and

2010 Mathematics Subject Classification. Primary: 46B42, 46E25, 54D15. Secondary: 46A40, 54D10, 54G05.

Key words and phrases. vector lattices, continuous functions, separation axioms, bands and projection bands, order continuity, un-convergence.

The first author acknowledges the financial support from the Slovenian Research Agency (research core funding No. P1-0222). The second author acknowledges the financial support from the Slovenian Research Agency (research core funding No. P1-0292, J1-8131 and J1-7025). 
$A \subseteq X$ is closed, order continuity of $\Phi_{A}$ is equivalent to $A=\overline{\operatorname{Int} A}$ (see Theorem 3.4).

Although order properties of $C(X)$ where $X$ is a compact Hausdorff space can be found in the main textbooks on vector and Banach lattices, results for $C_{b}(X)$ and $C(X)$ where $X$ is "just" Hausdorff seem to be left out of the main literature. Our second goal is to provide an elementary characterization of closed ideals, bands and projection bands of Banach lattices $C_{b}(X)$ and $C_{0}(X)$ through closed sets of $X$ where $X$ is Hausdorff and locally compact, respectively. The main tools that we use are order continuity of the restriction operator $\Phi_{A}$ and the embedding of $X$ into the compactification $X^{+}$or $\beta X$ where $X$ is locally compact Hausdorff space or $T_{3 \frac{1}{2}}$, respectively. There is also a way how to study $C_{b}(X)$ where $X$ is just Hausdorff.

If $X$ is $T_{3 \frac{1}{2}}$, then bands and projection bands have desired characterizations, and when $X$ is only Hausdorff, desired characterizations can be obtained only for projection bands (see Example 6.4).

In Section 7 we consider the so-called problem of "lifting un-convergence" [KMT17, Question 4.7]. An application of results from Section 4 and Section 5 provides a positive answer in the special case of Banach lattices $C_{0}(X)$ and $C_{b}(X)$.

\section{Preliminaries}

Let $E$ be a vector lattice. A vector subspace $Y$ of $E$ is an $\boldsymbol{i d e a l}$ or an order ideal whenever $0 \leq|x| \leq|y|$ and $y \in Y$ imply $x \in Y$. A band $B$ is an ideal with the property that $0 \leq x_{\alpha} \nearrow x$ and $x_{\alpha} \in B$ for each $\alpha$ imply $x \in B$. Here, the notation $x_{\alpha} \nearrow x$ means that the net $\left(x_{\alpha}\right)$ is increasing towards its supremum $x$. In the literature, bands are sometimes referred to as order closed ideals. For a given set $A \subseteq E$ we denote the set $\{x \in E:|x| \wedge|a|=0$ for all $a \in A\}$ by $A^{d}$. The set $A^{d}$ is called the disjoint complement of $A$ in $E$. It turns out that $A^{d}$ is always a band in $E$. If a band $B$ satisfies $E=B \oplus B^{d}$, then $B$ is called a projection band. If every band in $E$ is a projection band, then $E$ is said to have the projection property. A vector lattice is said to be order complete whenever nonempty bounded above sets have suprema. It is well-known that order complete vector lattices have 
the projection property. The spaces $L_{p}(\mu)$ are always order complete whenever $0<p<\infty$. If $\mu$ is $\sigma$-finite, then $L_{0}(\mu)$ and $L_{\infty}(\mu)$ are also order complete. On the other hand, the lattice $C(X)$ is rarely order complete. If $\left(f_{\alpha}\right)$ is an increasing net in $C(X)$ that is bounded above, then $f=\sup _{\alpha} f_{\alpha}$ exists in the vector lattice of all functions on $X$. If $f$ is continuous, then $f_{\alpha} \nearrow f$ in $C(X)$.

In this paper we are concerned with algebras/lattices of continuous functions on a topological space $X$. For a subset $A$ of $X$ we will denote by $\operatorname{Int}_{X} A$ and $\mathrm{Cl}_{X} A$ the interior and the closure of $A$ in $X$, respectively. If there is no confusion in which space the interior and the closure are taken, we will simply write $\operatorname{Int} A$ and $\bar{A}$ instead of $\operatorname{Int}_{A} X$ and $\mathrm{Cl}_{X} A$, respectively. A topological space is said to be completely regular if for every closed set $F$ in $X$ and $x \in X \backslash F$ there exists a continuous function $f: X \rightarrow[0,1]$ such that $f(x)=1$ and $\left.f\right|_{F} \equiv 0$. If $X$ is completely regular and Hausdorff, we say that $X$ is $T_{3 \frac{1}{2}}$ and we write $X \in T_{3 \frac{1}{2}}$ for short. It is well-known that locally compact Hausdorff spaces are $T_{3 \frac{1}{2}}$ (see e.g. Dug66, Theorem XI. 6.4]). A space $X$ is said to be regular whenever a point and a closed set which does not contain this point are contained in disjoint open sets. If disjoint closed sets of $X$ are contained in disjoint open sets, then $X$ is said to be $\boldsymbol{n o r m a l}$.

If not otherwise stated, all functions are assumed to be continuous. Since real function rings (resp. algebras) $C(X), C_{b}(X)$ and $C_{0}(X)$ are also lattices, we introduce the following notation to distinguish between algebraic and lattice notions of ideals. An algebra ideal is called an $\boldsymbol{a}$-ideal and an order ideal is called an o-ideal. Since all constant functions on $X$ are in $C_{b}(X)$ (resp. $C(X)$ ), the class of ring ideals in $C_{b}(X)$ (resp. $C(X)$ ) coincides with the class of $a$-ideals. An order or an algebra ideal $J$ in $C(X)$ is called fixed whenever all functions from $J$ vanish on a nonempty set. The ideals that are not fixed are called free. Fixed ideals play an important role in the characterization of compact Hausdorff spaces among $T_{3 \frac{1}{2}}$-spaces. For the proof of the following Theorem see [GJ76, Theorem 4.11].

Theorem 2.1. For $X \in T_{3 \frac{1}{2}}$ the following assertions are equivalent.

(a) $X$ is compact. 
(b) Every a-ideal in $C_{b}(X)$ is fixed.

(c) Every maximal a-ideal in $C_{b}(X)$ is fixed.

Special examples of fixed ideals are the ideals of the form

$$
J_{F}(X):=\left\{f \in C(X):\left.f\right|_{F} \equiv 0\right\}
$$

for some subset $F$ of $X$. Obviously $J_{F}(X)$ is an $a$-ideal and an $o$-ideal which is closed in $C(X)$ in the topology of pointwise convergence. It should be obvious that we always have $J_{F}(X)=J_{\bar{F}}(X)$. For $F \subseteq X$ we define $J_{F}^{0}(X)=\left\{f \in C_{0}(X):\left.f\right|_{F} \equiv 0\right\}$ and $J_{F}^{b}(X)=\left\{f \in C_{b}(X)\right.$ : $\left.\left.f\right|_{F} \equiv 0\right\}$.

If $X$ is a compact Hausdorff space there exists a characterization of closed ideals, bands and projection bands in $C(X)$. We start with a characterization of closed ideals (see e.g. [MN91, Proposition 2.1.9]).

Theorem 2.2. For every closed subspace $J$ of $C(X)$ where $X$ is a compact Hausdorff space the following assertions are equivalent.

(a) $J$ is an a-ideal.

(b) $J$ is an o-ideal.

(c) $J=J_{F}(X)$ for some closed set $F \subseteq X$.

When $X$ is compact and Hausdorff, the class of closed $a$-ideals in $C(X)$ coincides with the class of closed $o$-ideals; from now on in the case when $X$ is compact and Hausdorff we will call them closed ideals for short.

Bands and projection bands are characterized as follows (see e.g. [MN91, Corollary 2.1.10]).

Theorem 2.3. For a subspace $B$ of $C(X)$ where $X$ is a compact Hausdorff space the following assertions hold.

(a) $B$ is a band iff $B=J_{F}(X)$ for some set $F \subseteq X$ which is a closure of some open set.

(b) $B$ is a projection band iff $B=J_{F}(X)$ for some clopen set $F \subseteq$ $X$.

It should be noted that a closed set which is a closure of some open set is the closure of its interior. 
For unexplained facts about vector lattices and operators acting on them we refer the reader to [LZ71].

\section{ORDER CONTINUITY AND COMPLETELY REGULAR SPACES}

Complete regularity of the space basically means that points can be separated from closed sets by continuous functions. If we can separate only points by continuous functions, i.e., for different points $x, y \in X$ there is a continuous function $f: X \rightarrow[0,1]$ with $f(x)=0$ and $f(y)=$ 1 , then $X$ is called functionally Hausdorff.

If $X$ is not functionally Hausdorff, then there exist distinct $x, y \in$ $X$ such that $f(x)=f(y)$ for all $f \in C(X)$. In this case we have $J_{\{x\}}(X)=J_{\{y\}}(X)$, so that the mapping $x \mapsto J_{\{x\}}(X)$ is not one-toone. Similarly, if $X \in T_{3 \frac{1}{2}}$, then for closed sets $F$ and $G$ in $X$ we have $J_{F}(X)=J_{G}(X)$ iff $F=G$. Also, when $F$ is a closed set in $X \in T_{3 \frac{1}{2}}$, the disjoint complement of $J_{F}(X)$ satisfies $J_{F}(X)^{d}=J_{\overline{X \backslash F}}(X)$.

In this section we are interested in the so-called "restriction operator", defined as follows. If $A$ is a subset of $X$, then the restriction $\left.f\right|_{A}$ of $f \in C(X)$ is continuous on $A$. The mapping $\Phi_{A}: C(X) \rightarrow C(A)$, defined as $\Phi_{A}(f)=\left.f\right|_{A}$, is called the restriction operator. Also, if $f \in C_{b}(X)$, then $\left.f\right|_{A} \in C_{b}(A)$ and the restriction operator is a contractive lattice homomorphism with respect to the $\|\cdot\|_{\infty}$ norms on $C_{b}(X)$ and $C_{b}(A)$. By Tietze's extension theorem, $\Phi_{A}$ is surjective for all closed subsets $A \subseteq X$ iff $X$ is normal.

Suppose $f_{\alpha} \nearrow f$ in $C(X)$. Then $f$ is not necessarily the pointwise limit of the net $\left(f_{\alpha}\right)$. In general the set of all $x \in X$ with $f_{\alpha}(x) \rightarrow f(x)$ can be empty even when $X$ is metrizable (in particular when $X \in T_{3 \frac{1}{2}}$ ), as it is shown in the following example.

Example 3.1. Let $X=\mathbb{Q} \cap[0,1]$. Enumerate elements in $X$ as $\left(q_{n}\right)$ such that $q_{0}=0$ and $q_{1}=1$. For each $n \in \mathbb{N}$ we define piece-wise linear functions $f_{n}: \mathbb{Q} \rightarrow[0,1]$ inductively. Let $f_{1}=0$. For each $n \geq 2$ we define $l_{n}:=\max \left\{q_{k}<q_{n}: k=0, \ldots, n-1\right\}$ and $r_{n}:=\min \left\{q_{k}>q_{n}\right.$ : $k=0, \ldots, n-1\}$. The function $f_{n}$ is defined such that it agrees with $f_{n-1}$ on $X \backslash\left(l_{n}, r_{n}\right), f_{n}\left(q_{n}\right)=1-\frac{1}{n}$ and $f_{n}$ is linear on $\left[l_{n}, q_{n}\right] \cap \mathbb{Q}$ and $\left[q_{n}, r_{n}\right] \cap \mathbb{Q}$. For every $q_{k} \in X$ we have $\lim _{n \rightarrow \infty} f_{n}\left(q_{k}\right)=1-\frac{1}{k}<1$. We 
claim that $f_{n} \nearrow \mathbb{1}$. By the construction we have $f_{n} \leq \mathbb{1}$. Suppose that $f_{n} \leq f$ for some $f \in C(X)$ and suppose that there exists $x \in X$ such that $f(x)=c<1$. There exists an open set $U$ such that $f(y)<\frac{1+c}{2}$ for all $y \in U$. But there exists $n$ such that $q_{n} \in U$ and $1-\frac{1}{n}>\frac{1+c}{2}$, hence $f_{n}\left(q_{n}\right)=1-\frac{1}{n}>f\left(q_{n}\right)$, which is a contradiction.

In the case when $X$ is a locally compact Hausdorff space, the set $\left\{x \in X: f_{\alpha}(x) \rightarrow f(x)\right\}$ is dense in $X:$

Proposition 3.2. Let $0 \leq f_{\alpha} \nearrow$ in $C(X)$ and $f_{\alpha} \leq f$ for all $\alpha$.

(a) If the set $\left\{x \in X: f_{\alpha}(x) \rightarrow f(x)\right\}$ is dense in $X$, then $f_{\alpha} \nearrow f$.

(b) If $X$ is a locally compact Hausdorff space and $f_{\alpha} \nearrow f$, then the set $\left\{x \in X: f_{\alpha}(x) \rightarrow f(x)\right\}$ is dense in $X$.

Proof. (a) If $f_{\alpha} \leq g$ for all $\alpha$, then $f(x) \leq g(x)$ on a dense subset of $X$. By continuity we conclude $f \leq g$.

(b) We claim that for every open subset $V \subset X$ and $\epsilon>0$ there exist $\alpha$ and $x \in V$ such that $f(x)-f_{\alpha}(x)<\epsilon$. If that were not the case, then $f(x)-f_{\alpha}(x) \geq \epsilon$ for all $\alpha$ and $x \in V$. Pick any nonzero continuous function $\varphi: X \rightarrow\left[0, \frac{\varepsilon}{2}\right]$ such that $\varphi(X \backslash V)=0$. Then $f_{\alpha} \leq f-\varphi$ for all $\alpha$, which contradicts $f_{\alpha} \nearrow f$.

Let $U \subset X$ be an open set. We will show that $U \cap Q \neq \emptyset$, where $Q=\left\{x \in X: f_{\alpha}(x) \rightarrow f(x)\right\}$. Because $X$ is locally compact there exists an open set $U_{0}$ such that $\bar{U}_{0} \subset U$ is compact. Suppose we have already defined $U_{n-1}$. By the previous paragraph there exists $\alpha$ such that $U_{n}:=\left\{x \in U_{n-1}: f(x)-f_{\alpha}(x)<\frac{1}{n}\right\} \neq \emptyset$. Since $\bar{U}_{n} \subseteq \bar{U}_{n-1}$ is compact, we have $\bigcap_{n=0}^{\infty} \bar{U}_{n} \neq \emptyset$. Hence for every $x \in \bigcap_{n=0}^{\infty} \bar{U}_{n} \subseteq U$ we have $f(x)=\sup _{\alpha} f_{\alpha}(x)$, which proves $x \in U \cap Q$.

Suppose again $f_{\alpha} \nearrow f$ in $C(X)$. Then for each set $A \subseteq X$ we have $\left.f_{\alpha}\right|_{A} \nearrow$ and $\left.f_{\alpha}\right|_{A} \leq\left. f\right|_{A}$ for each $\alpha$. Proposition 3.2 shows that $\left.\left.f_{\alpha}\right|_{A} \nearrow f\right|_{A}$, when $A$ is an open subset of a locally compact Hausdorff space $X$. In general we cannot expect $\left.\left.f_{\alpha}\right|_{A} \nearrow f\right|_{A}$ since $\Phi_{A}$ is not order continuous. We will prove that for a closed set $A \subseteq X \in T_{3 \frac{1}{2}}$, the operator $\Phi_{A}: C(X) \rightarrow C(A)$ is order continuous iff $A=\overline{\operatorname{Int} A}$ (see Theorem 3.4. To prove this result we need the following proposition.

Proposition 3.3. Let $A$ be an open subset of $X \in T_{3 \frac{1}{2}}$. 
(a) If $f_{\alpha} \nearrow f$ in $C(X)$, then $\left.\left.f_{\alpha}\right|_{A} \nearrow f\right|_{A}$ in $C(A)$.

(b) If $f_{\alpha} \nearrow f$ in $C_{b}(X)$, then $\left.\left.f_{\alpha}\right|_{A} \nearrow f\right|_{A}$ in $C_{b}(A)$.

Proof. (a) Let $f_{\alpha} \nearrow f$ in $C(X)$. Suppose there exists $g \in C(A)$ such that $\left.f_{\alpha}\right|_{A} \leq g<\left.f\right|_{A}$ for all $\alpha$. There exists $x_{0} \in A$ such that $g\left(x_{0}\right)<$ $f\left(x_{0}\right)$ and because $f$ and $g$ are continuous and $X$ is regular, there exists an open set $U \subseteq \mathrm{Cl}_{X}(U) \subseteq A$ such that $g(x)<f(x)$ for all $x \in U$. Because $X \in T_{3 \frac{1}{2}}$ there exists $\lambda: X \rightarrow[0,1]$ such that $\lambda\left(x_{0}\right)=1$ and $\lambda(X \backslash U)=0$. We define a function $\lambda g: X \rightarrow \mathbb{R}$ as

$$
(\lambda g)(x)=\left\{\begin{array}{cl}
\lambda(x) g(x) & : x \in A \\
0 & : \quad x \in X \backslash \mathrm{Cl}_{X} U
\end{array} .\right.
$$

Since $\left\{A, X \backslash \mathrm{Cl}_{X}(U)\right\}$ is an open cover of $X$ and $\lambda \equiv 0$ on $X \backslash \mathrm{Cl}_{X} U$, we conclude that $\lambda g$ is continuous on $X$. Then $h: X \rightarrow \mathbb{R}$, defined by $h(x)=(\lambda g)(x)+(1-\lambda(x)) f(x)$ is continuous and $f_{\alpha} \leq h<f$ for all $\alpha$. This is a contradiction, as $f_{\alpha} \nearrow f$.

(b) Suppose $f_{\alpha} \nearrow f$ in $C_{b}(X)$. Since $C_{b}(X)$ is an order ideal in $C(X)$, we have $f_{\alpha} \nearrow f$ in $C(X)$, so that by (a) we have $\left.\left.f_{\alpha}\right|_{A} \nearrow f\right|_{A}$ in $C(X)$ and so in $C_{b}(X)$.

Proposition 3.3 shows that the condition $X \in T_{3 \frac{1}{2}}$ is sufficient to imply that $\Phi_{A}: C(X) \rightarrow C(A)$ is order continuous for every open set $A$ in $X$. However, Example 6.5 will show that this condition is not necessary.

Theorem 3.4. Let $A$ be a closed subset of $X \in T_{3 \frac{1}{2}}$. The following are equivalent:

(a) $A=\overline{\operatorname{Int} A}$.

(b) If $f_{\alpha} \nearrow f$ in $C(X)$, then $\left.\left.f_{\alpha}\right|_{A} \nearrow f\right|_{A}$ in $C(A)$.

(c) If $f_{\alpha} \nearrow f$ in $C_{b}(X)$, then $\left.\left.f_{\alpha}\right|_{A} \nearrow f\right|_{A}$ in $C_{b}(A)$.

Proof. (a) $\Rightarrow$ (b) Suppose $A=\overline{\operatorname{Int} A}$ and let $f_{\alpha} \nearrow f$ in $C(X)$. Let $g: A \rightarrow \mathbb{R}$ be such that $\left.f_{\alpha}\right|_{A} \leq g$ for all $\alpha$. By Proposition 3.3. $\left.\left.f_{\alpha}\right|_{\operatorname{Int} A} \nearrow f\right|_{\operatorname{Int} A}$ in $C(\operatorname{Int} A)$, hence $\left.g\right|_{\operatorname{Int} A} \geq\left. f\right|_{\operatorname{Int} A}$. Because of continuity of $f$ and $g$ (on $A$ ) and $A=\overline{\operatorname{Int} A}$ we get $g \geq\left. f\right|_{A}$.

(b) $\Rightarrow$ (a) Suppose there exists $x_{0} \in A \backslash \overline{\operatorname{Int} A}$. Because $X \in T_{3 \frac{1}{2}}$, there exists $\widetilde{\psi}: X \rightarrow[-1,1]$ such that $\widetilde{\psi}\left(x_{0}\right)=-1$ and $\widetilde{\psi}(\overline{\operatorname{Int} A})=1$. 
Then $U=\widetilde{\psi}^{-1}([-1,0)) \cap A$ is an open set in $A$. Because $A$ is closed, $\bar{U} \subseteq A$ and $\bar{U} \subseteq \widetilde{\psi}^{-1}([-1,0])$, hence $x_{0} \in U \subseteq \bar{U} \subseteq A \backslash \overline{\operatorname{Int} A}$. Let $\mathcal{M}=\left\{f: X \rightarrow[0,1]:\left.f\right|_{\bar{U}} \equiv 0,\left.f\right|_{\overline{\operatorname{Int} A}} \equiv 1\right\}$. The map $\psi: X \rightarrow[0,1]$, defined as $\psi=\max \{\widetilde{\psi}, 0\}$ is in $\mathcal{M}$, therefore $\mathcal{M}$ is nonempty.

Claim 1: If $f \leq g$ for all $f \in \mathcal{M}$, then $g(x) \geq 1$ for all $x \in X \backslash \bar{U}$. Indeed, pick $x \notin \bar{U}$. Because $X \in T_{3 \frac{1}{2}}$ there exists $\varphi: X \rightarrow[0,1]$ such that $\varphi \equiv 0$ on $\bar{U}$ and $\varphi(x)=1$. Then $f:=\min \{\varphi+\psi, \mathbb{1}\} \in \mathcal{M}$ and $f(x)=1$, which implies $g(x) \geq f(x)=1$.

Claim 2: The set $X \backslash \bar{U}$ is dense in $X$. Indeed, otherwise there exists an open set $V \subseteq X$ such that $V \cap(X \backslash \bar{U})=\emptyset$. Then $V \subseteq \bar{U} \subseteq$ $A \backslash \overline{\operatorname{Int} A}$. This is a contradiction, since from $V \subseteq A$ and $V$ open follows $V \subseteq \operatorname{Int} A$.

Let us show that $(f)_{f \in \mathcal{M}} \nearrow \mathbb{1}$ in $C(X)$. Suppose some function $g: X \rightarrow \mathbb{R}$ satisfies $f \leq g$ for all $f \in \mathcal{M}$. Claim 1 yields that $g \geq \mathbb{1}$ on $X \backslash \bar{U}$ and since by Claim 2 the set $X \backslash \bar{U}$ is dense in $X$, we have $g \geq \mathbb{1}$ on $X$. Therefore, $(f)_{f \in \mathcal{M}} \nearrow \mathbb{1}$ in $C(X)$.

If $\left(\left.f\right|_{A}\right)_{f \in \mathcal{M}} \nearrow \mathbb{1}$ in $C(A)$, by Proposition $3.3\left(\left.f\right|_{U}\right)_{f \in \mathcal{M}} \nearrow \mathbb{1}$. But $\left.f\right|_{U} \equiv 0$ for all $f \in \mathcal{M}$.

(b) $\Rightarrow(\mathrm{c})$ Suppose $f_{\alpha} \nearrow f$ in $C_{b}(X)$. Then $f_{\alpha} \nearrow f$ in $C(X)$, so that $\left.\left.f_{\alpha}\right|_{A} \nearrow f\right|_{A}$ in $C(A)$. Since $\left.f\right|_{A}$ is bounded, we have $\left.\left.f_{\alpha}\right|_{A} \nearrow f\right|_{A}$ in $C_{b}(A)$.

(c) $\Rightarrow(\mathrm{b})$ Since for each $0 \leq g \in C(X)$ we have $g \wedge n \mathbb{1} \nearrow_{n} g$ and since for each $n \in \mathbb{N}$ we have $\left.\left.f_{\alpha}\right|_{A} \wedge n \mathbb{1} \nearrow_{\alpha} f\right|_{A} \wedge n \mathbb{1}$, we conclude

$$
\begin{aligned}
\left.f\right|_{A} & =\sup _{n \in \mathbb{N}}\left(\left.f\right|_{A} \wedge n \mathbb{1}\right)=\sup _{n \in \mathbb{N}} \sup _{\alpha}\left(\left.f_{\alpha}\right|_{A} \wedge n \mathbb{1}\right) \\
& =\sup _{\alpha} \sup _{n \in \mathbb{N}}\left(\left.f_{\alpha}\right|_{A} \wedge n \mathbb{1}\right)=\left.\sup _{n \in \mathbb{N}} f_{\alpha}\right|_{A} .
\end{aligned}
$$

Corollary 3.5. Let $A$ be a closed subset in $X \in T_{3 \frac{1}{2}}$. Then the restriction operator $\Phi_{A}$ is order continuous iff $A=\overline{\operatorname{Int} A}$.

Suppose now that $X$ is compact and Hausdorff; hence normal and so $X \in T_{3 \frac{1}{2}}$. If $A$ is closed in $X$, then the kernel of $\Phi_{A}: C(X) \rightarrow C(A)$ is precisely the fixed ideal $J_{A}(X)$. In this special case, by Theorem 2.3 and Corollary 3.5 , the restriction operator $\Phi_{A}$ is order continuous iff $J_{A}(X)$ is a band in $C(X)$. 
When $X$ is merely normal, the restriction operator $\Phi_{A}$ is surjective. By [LZ71, Theorem 18.13] $\Phi_{A}: C(X) \rightarrow C(A)$ is order continuous iff ker $\Phi_{A}=J_{A}(X)$ is a band in $C(X)$. In Corollary 4.5 we will prove that for $X \in T_{3 \frac{1}{2}}$ bands in $C(X)$ are precisely closed $o$-ideals in $C(X)$ of the form $J_{A}(X)$ where $A$ is a closed set satisfying $A=\overline{\operatorname{Int} A}$.

If we assume only that $X \in T_{3 \frac{1}{2}}$, then the restriction operator $\Phi_{A}$ is not surjective in general. When $A=\overline{\operatorname{Int} A}$, then Corollary 4.5 implies $J_{A}(X)$ is a band in $C(X)$ and $\Phi_{A}: C(X) \rightarrow \operatorname{Ran} \Phi_{A} \subseteq C(A)$ is an order continuous lattice homomorphism by [LZ71, Theorem 18.13]. Therefore, if $f_{\alpha} \nearrow f$ in $C(X)$, then $\left.\left.f_{\alpha}\right|_{A} \nearrow f\right|_{A}$ in the sublattice $\operatorname{Ran} \Phi_{A}$. Since $\operatorname{Ran} \Phi_{A}$ is order dense in $C(A)$ (see Proposition 3.6 below), we have $\left.\left.f_{\alpha}\right|_{A} \nearrow f\right|_{A}$ in $C(A)$.

Proposition 3.6. Let $A$ be a subset of $X \in T_{3 \frac{1}{2}}$ and let $\Phi_{A}: C(X) \rightarrow$ $C(A)$ be the restriction operator. Then $\operatorname{Ran} \Phi_{A}$ is order dense in $C(A)$.

Proof. Pick a nonzero nonnegative function $f \in C(A)$. Let $a \in A$ be such that $f(a)>0$. Because $f$ is continuous, there exists an open set $U$ in $A$ such that $f(x)>\frac{f(a)}{2}$ for all $x \in U$. Let $V$ be open in $X$ such that $V \cap A=U$. Because $X \in T_{3 \frac{1}{2}}$ there exists $g: X \rightarrow\left[0, \frac{f(a)}{2}\right]$ such that $g(a)=\frac{f(a)}{2}$ and $g \equiv 0$ on $X \backslash V$. For $x \in U$ we have $f(x)>\frac{f(a)}{2} \geq g(x)$, and for $x \in A \backslash U$ we have $f(x) \geq 0=g(x)$. Hence $0 \leq\left. g\right|_{A} \leq f$.

\section{Bands in $C(X)$ : Completely Regular Case}

In this section we are interested in extending Theorem 2.2 and Theorem 2.3 to the case of the algebra $C(X)$ where $X \in T_{3 \frac{1}{2}}$. It turns out that the class of all closed $a$-ideals in $C_{b}(X)$ again coincides with the class of all closed $o$-ideals (see Remark 4.1). From now on we will simply call them closed ideals. To have a nice characterization of closed ideals of $C_{b}(X)$ in terms of closed subsets of $X$ is too optimistic even when $X \in T_{3 \frac{1}{2}}$. If for some $X \in T_{3 \frac{1}{2}}$ every closed ideal in $C_{b}(X)$ is of the form $J_{F}^{b}(X)$ for some closed set $F$ in $X$, then every closed ideal in $C_{b}(X)$ is fixed, so that by Theorem $2.1 X$ is compact. Hence, if $X$ is a noncompact space, then there exist closed ideals in $C_{b}(X)$ which are not of the form $J_{F}^{b}(X)$ for any closed set $F$ in $X$. However, if $X \in T_{3 \frac{1}{2}}$ then bands and projection bands in $C_{b}(X)$ and $C(X)$ have 
similar characterizations as bands in $C(K)$-spaces (see Theorem 4.4 and Corollary 4.5). If $X$ is merely Hausdorff, then projection bands in $C_{b}(X)$ and $C(X)$ have a similar characterization as those in $C(K)$ spaces (see Theorem 6.3). Unfortunately, Example6.4 shows that there exists a functionally Hausdorff space $X$ and a band $J_{F}^{b}(X)$ in $C_{b}(X)$ with $\overline{\operatorname{Int} F} \neq F$. However, it can happen that there exists a closed subset $G$ of $X$ with $G=\overline{\operatorname{Int} G}$ and $J_{G}^{b}(X)=J_{F}^{b}(X)$.

Suppose $X \in T_{3 \frac{1}{2}}$. There exists a compact Hausdorff space $\beta X$ which contains $X$ as a dense subspace, and has the property that every function $f \in C_{b}(X)$ can be uniquely extended to the continuous function $f^{\beta} \in C(\beta X)$. The space $\beta X$ is called the Stone- $\check{C}$ ech compactification of $X$. Furthermore, by [ABo06, Theorem 3.70], $X$ is an open subset of $\beta X$ whenever $X$ is locally compact and Hausdorff. The mapping $\beta: C_{b}(X) \rightarrow C(\beta X)$, defined as $\beta(f)=f^{\beta}$ is an isometric isomorphism of Banach algebras. By [GJ76, Theorem 1.6] the mapping $\beta$ is also a lattice isomorphism. For more details on the Stone-Čech compactification we refer the reader to [HS12.

Remark 4.1. Having in mind that $C_{b}(X)$ is isometrically lattice and algebra isomorphic to $C(\beta X)$, a subset $J$ of $C_{b}(X)$ is a closed $a$-ideal (resp., o-ideal, band, projection band) iff its isomorphic copy in $C(\beta X)$ is a closed $a$-ideal (resp., $o$-ideal, band, projection band). Applying Theorem 2.2 we see that the class of all closed $a$-ideals in $C_{b}(X)$ again coincides with the class of all closed $o$-ideals.

As we already mentioned, if $X$ is not compact, by Theorem 2.1 there exists a closed ideal $J$ in $C_{b}(X)$ which is not fixed. However, there exists a closed subset $F \subseteq \beta X$ such that $J=\left\{\left.f\right|_{X}: f \in J_{F}(\beta X)\right\}$. Since $J$ is not fixed, $F$ is not a subset of $X$. Although the description of $J$ is sufficient to have some information on closed ideals of $C_{b}(X)$, it is not the best one since $F$ is a subset of $\beta X$ and not of $X$. If $J$ is a band or a projection band in $C_{b}(X)$, then $J$ is fixed (see Theorem 4.4). Before we can obtain the desired characterization, we need the following two results.

Lemma 4.2. Let $F$ be a closed set in $X \in T_{3 \frac{1}{2}}$. Then $\beta\left(J_{F}^{b}(X)\right)=$ $J_{\bar{F}}(\beta X)$ where $\bar{F}$ denotes the closure of $F$ in $\beta X$. 
Proof. If $f \in J_{F}^{b}(X)$, then $f \equiv 0$ on $F$, and hence by continuity $f^{\beta} \equiv 0$ on $\bar{F}$. For the opposite inclusion, pick $f^{\beta} \in J_{\bar{F}}(\beta X)$. Then $f \equiv 0$ on $F$, so that $f^{\beta} \in \beta\left(J_{F}^{b}(X)\right)$.

The following proposition will be used in the proof of Theorem 4.4. The proof is straightforward and it is left for the reader as an exercise.

Proposition 4.3. Let $X$ be a dense subset of a topological space $Y$. If a closed set $F \subseteq Y$ satisfies $F=\mathrm{Cl}_{Y} \operatorname{Int}_{Y} F$, then the closed set $F \cap X$ in $X$ satisfies $F \cap X=\mathrm{Cl}_{X} \operatorname{Int}_{X}(F \cap X)$.

Theorem 4.4. Let $X \in T_{3 \frac{1}{2}}$ and let $B$ be a subspace in $C_{b}(X)$.

(a) $B$ is a band in $C_{b}(X)$ iff $B=J_{F}^{b}(X)$ for some closed set $F$ in $X$ with $F=\overline{\operatorname{Int} F}$.

(b) $B$ is a projection band iff $B=J_{F}^{b}(X)$ for some clopen subset $F$ of $X$.

Proof. (a) Suppose $B$ is a band in $C_{b}(X)$. By Theorem 2.3 there exists a closed set $F \subseteq \beta X$ such that $F=\mathrm{Cl}_{\beta X} \operatorname{Int}_{\beta X} F$ and $\beta(B)=J_{F}(\beta X)$.

We claim that $B=J_{F \cap X}^{b}(X)$. Let us denote $B^{\prime}=J_{F \cap X}^{b}(X)$. Then $\beta\left(B^{\prime}\right)=J_{\mathrm{Cl}_{\beta X}(F \cap X)}(\beta X)$. By a standard topological argument we have $\mathrm{Cl}_{\beta X}(F \cap X)=F$, so that $\beta\left(B^{\prime}\right)=\beta(B)$. Hence, $B^{\prime}=B$ as claimed.

To finish the proof of the forward implication we apply Proposition 4.3 for $Y=\beta X$ to obtain $F \cap X=\mathrm{Cl}_{X} \operatorname{Int}_{X}(F \cap X)$.

Suppose now that $B=J_{F}^{b}(X)$ and $F$ is the closure of its interior in $X$. If $0 \leq f_{\alpha} \nearrow f$ in $C_{b}(X)$ and $f_{\alpha} \in J_{F}^{b}(X)$ for each $\alpha$, then $\left.\left.f_{\alpha}\right|_{\operatorname{Int} F} \nearrow f\right|_{\operatorname{Int} F}$, by Proposition 3.3. Since $f_{\alpha} \equiv 0$ on Int $F$, we have $f \equiv 0$ on $\operatorname{Int} F$, so that by continuity of $f$ we conclude $f \equiv 0$ on $\overline{\operatorname{Int} F}=F$. Hence, $f \in J_{F}^{b}(X)$ and $B=J_{F}^{b}(X)$ is a band in $C_{b}(X)$.

(b) Suppose that $J$ is a projection band in $C_{b}(X)$. By (a) we have $J=J_{F}^{b}(X)$ for some closed set $F$ in $X$. We claim $X \backslash F$ is closed in $X$. Otherwise there is $x_{0} \in F \cap \overline{X \backslash F}$. Pick any function $f \in C_{b}(X)$ with $f(x)=1$. Since $J$ is a projection band, we have $C_{b}(X)=J_{F}^{b}(X) \oplus$ $J_{F}^{b}(X)^{d}=J_{F}^{b}(X) \oplus J_{\overline{X \backslash F}}^{b}(X)$. Hence, $f=f_{1}+f_{2}$ for some $f_{1} \in J_{F}^{b}(X)$ and $f_{2} \in J_{X \backslash F}^{b}(X)$, so that $f\left(x_{0}\right)=0$. This contradiction shows $X \backslash F$ is closed. 
For the converse, assume $F$ is clopen in $X$. Then the characteristic functions $\chi_{F}$ and $\chi_{X \backslash F}$ are continuous on $X$. Since each function $f \in$ $C_{b}(X)$ can be written as $f=f \cdot \chi_{X \backslash F}+f \cdot \chi_{F} \in J_{F}^{b}(X) \oplus J_{F}^{b}(X)^{d}$, we conclude that $J_{F}^{b}(X)$ is a projection band in $C_{b}(X)$.

The preceding theorem allows us to characterize bands and projection bands in $C(X)$ where $X \in T_{3 \frac{1}{2}}$.

Corollary 4.5. For a subspace $B$ of $C(X)$ where $X \in T_{3 \frac{1}{2}}$ the following assertions hold.

(a) $B$ is a band iff $B=J_{F}(X)$ for some closed set $F$ in $X$ with $F=\overline{\operatorname{Int} F}$.

(b) $B$ is a projection band iff $B=J_{F}(X)$ for some clopen set $F$ in $X$.

Proof. (a) If $B$ is a band in $C(X)$, then it is easy to see that $B^{\prime}:=$ $B \cap C_{b}(X)$ is a band in $C_{b}(X)$. By Theorem 4.4 there exists a closed subset $F$ in $X$ with $F=\overline{\operatorname{Int} F}$ and $B^{\prime}=J_{F}^{b}(X)$. We claim $B=J_{F}(X)$. To this end, pick $0 \leq f \in B$. Then $f \wedge \mathbb{1} \in B^{\prime}=J_{F}^{b}(X)$, so that $f \equiv 0$ on $F$. Suppose now $0 \leq f \in J_{F}(X)$. Then for each $n$ we have $f \wedge n \mathbb{1} \in J_{F}^{b}(X)=B^{\prime} \subseteq B$. Since $B$ is a band and $f \wedge n \mathbb{1} \nearrow f$, we conclude $f \in B$.

Suppose now $B=J_{F}(X)$ for some closed set $F$ in $X$ with $F=\overline{\operatorname{Int} F}$. Pick a net $\left(f_{\alpha}\right)$ in $B$ with $0 \leq f_{\alpha} \nearrow f$. Then $f_{\alpha} \wedge \mathbb{1} \nearrow f \wedge \mathbb{1}$ and since by Theorem $4.4 J_{F}^{b}(X)$ is a band in $C_{b}(X)$, we conclude $f \wedge \mathbb{1} \in J_{F}^{b}(X)$. This implies $f \equiv 0$ on $F$, so that $f \in J_{F}(X)$.

(b) The proof is similar to the proof of Theorem 4.4(b) and is therefore omitted.

\section{BAnds in $C_{0}(X)$}

In this section we are interested in closed ideals of the Banach lattice algebra $C_{0}(X)$ where $X$ is a locally compact Hausdorff space. We characterize closed ideals, bands and projection bands of $C_{0}(X)$ (see Theorem 5.1 and Theorem 5.2. The characterizations are pretty much the same as the characterization of closed ideals, bands and projection bands of the algebra and vector lattice of all continuous functions on 
a given compact Hausdorff space (see Theorem 2.2 and Theorem 2.3). Our arguments presented in this section are based on the embedding of $X$ into its one-point compactification $X^{+}=X \cup\{\infty\}$. If $X$ is locally compact and Hausdorff, the embedding $X \hookrightarrow X^{+}$is an open mapping by Dug66, 8.3 p.245]. If $X$ is not compact, then it is an open and dense subset of $X^{+}$. If $X$ is compact, then $X$ is clopen in $X^{+}$and $\infty$ is isolated in $X^{+}$.

The restriction operator $\Phi_{X}: C\left(X^{+}\right) \rightarrow C(X)$ induces the algebra and lattice isometric isomorphism between $J_{\{\infty\}}\left(X^{+}\right)$in $C\left(X^{+}\right)$and the closed maximal lattice and algebra ideal $C_{0}(X)$. By an abuse of notation we will identify order and algebra ideals of $C_{0}(X)$ with their isometric isomorphic copies in $J_{\{\infty\}}\left(X^{+}\right)$. In particular, for any subset $F$ of $X$ we identify $J_{F}^{0}(X)$ with $J_{F \cup\{\infty\}}\left(X^{+}\right)$. Therefore, if $X$ is not compact, $C_{0}(X)$ which is identified with $J_{\{\infty\}}\left(X^{+}\right)$is not a band in $C\left(X^{+}\right)$, since $\{\infty\}$ is not the closure of its interior (see Theorem 2.3.).

As was already mentioned, closed ideals are characterized similarly as the closed ideals in $C(K)$-spaces.

Theorem 5.1. Let $X$ be a locally compact Hausdorff space and $J$ a closed subspace in $C_{0}(X)$. The following assertions are equivalent:

(a) $J$ is an a-ideal in $C_{0}(X)$.

(b) $J$ is an o-ideal in $C_{0}(X)$.

(c) $J=J_{F}^{0}(X)$ for some closed set $F$ in $X$.

Proof. When $X$ is compact the conclusion follows from Theorem 2.2, so that we may assume $X$ is not compact. By [Kan09, Theorem 1.4.6] (a) and (c) are equivalent. It is trivial that (c) implies (b).

To finish the proof we prove that (b) implies (a). Since $C_{0}(X)$ is isometrically lattice isomorphic to $J_{\{\infty\}}\left(X^{+}\right), J$ is isometrically lattice isomorphic to some closed o-ideal $J^{\prime}=J_{F^{\prime}}\left(X^{+}\right)$of $C\left(X^{+}\right)$for some closed subset $F^{\prime} \subseteq X^{+}$, which contains the point $\infty$. By Theorem 2.2 $J^{\prime}$ is also an $a$-ideal, from where it follows that $J=\left\{\left.f\right|_{X}: f \in J^{\prime}\right\}$ is an $a$-ideal in $C_{0}(X)$.

When $X$ is locally compact and Hausdorff, the class of closed $a$-ideals in $C_{0}(X)$ again coincides with the class of closed $o$-ideals; from now on we will call them closed ideals for short. 
We proceed by a characterization of bands and projection bands in $C_{0}(X)$. Our proof presented here relies on the fact that $X \in T_{3 \frac{1}{2}}$ whenever it is locally compact and Hausdorff.

Theorem 5.2. Let $X$ be a locally compact Hausdorff space and $J$ a subspace in $C_{0}(X)$.

(a) $J$ is a band iff $J=J_{F}^{0}(X)$ for some closed set $F$ in $X$ with $F=\overline{\operatorname{Int} F}$.

(b) $J$ is a projection band iff $J=J_{F}^{0}(X)$ for some clopen set $F$ in $X$.

Proof. (a) Suppose first that $J$ is a band in $C_{0}(X)$. By $\widetilde{J}$ we denote the band generated by $J$ in $C_{b}(X)$. By Theorem 4.4 there exists a closed subset $F$ of $X$ with $F=\overline{\operatorname{Int} F}$ such that $\widetilde{J}=J_{F}^{b}(X)$. We claim that $J=J_{F}^{0}(X)$. To prove this, it suffices to prove $J=\widetilde{J} \cap C_{0}(X)$.

Pick $f \in \widetilde{J} \cap C_{0}(X)$. Since $\widetilde{J}$ is the band generated by $J$ in $C_{b}(X)$, there is a net $\left(f_{\alpha}\right)$ in $J$ such that $0 \leq f_{\alpha} \nearrow|f|$ in $C_{b}(X)$. Since $|f| \in C_{0}(X)$, we conclude $0 \leq f_{\alpha} \nearrow|f|$ in $C_{0}(X)$. Now apply the fact that $J$ is a band in $C_{0}(X)$ to get $f \in J$. The reverse inclusion is obvious.

Suppose now that a subspace $J$ in $C_{0}(X)$ is of the form $J_{F}^{0}(X)$ for some closed set $F$ in $X$ with $F=\overline{\operatorname{Int} F}$. Then $J_{F}^{b}(X)$ is a band in $C_{b}(X)$ by Theorem 4.4. This implies that $J_{F}^{0}(X)=J_{F}^{b}(X) \cap C_{0}(X)$ is a band in $C_{0}(X)$.

(b) The proof is again omitted since it is very similar to the proof of Theorem 4.4(b).

Pick a closed set $F$ in $X$. Recall that we identify a closed ideal $J_{F}^{0}(X)$ in $C_{0}(X)$ with its isomorphic copy $J_{F \cup\{\infty\}}\left(X^{+}\right)$in $J_{\{\infty\}}\left(X^{+}\right) \subseteq$ $C\left(X^{+}\right)$. We are interested under which conditions on $F$ the closed ideal $J_{F \cup\{\infty\}}\left(X^{+}\right)$is a projection band in $C\left(X^{+}\right)$. In view of Theorem 5.2 one would think that a sufficient and necessary condition is that $F$ is clopen. However, this is not enough:

Corollary 5.3. Let $F$ be a closed subset of a locally compact Hausdorff space $X$. Then $J_{F}^{0}(X) \cong J_{F \cup\{\infty\}}\left(X^{+}\right)$is a projection band in $C\left(X^{+}\right)$ iff $X \backslash F$ is compact. 
Proof. Suppose first that $J_{F \cup\{\infty\}}\left(X^{+}\right)$is a projection band in $C\left(X^{+}\right)$. By Theorem 2.3(b) $F \cup\{\infty\}$ is clopen in $X^{+}$. Since $X \backslash F=X^{+} \backslash$ $(F \cup\{\infty\})$ is also closed in $X^{+}$, the set $X \backslash F$ is compact.

Suppose now that $X \backslash F$ is compact. Since $F \cup\{\infty\}$ is closed in $X^{+}$ and $X \backslash F$ is compact, $F \cup\{\infty\}$ is clopen in $X^{+}$. By Theorem 2.3(b) $J_{F}^{0}(X) \cong J_{F \cup\{\infty\}}\left(X^{+}\right)$is a projection band in $C\left(X^{+}\right)$.

\section{Bands in $C(X)$ : Hausdorff CASE}

In this section we are interested in extending Theorem 4.4 to the case when $X$ is only a Hausdorff space. It turns out that projection bands can be characterized similarly as in Theorem 4.4, while there is no such characterization for bands (see Example 6.4). To obtain our result for a given Hausdorff space $X$ we associate $X / \sim \in T_{3 \frac{1}{2}}$, such that $C_{b}(X)$ and $C_{b}(X / \sim)$ are lattice and algebra isometric isomorphic:

On $X$ we define an equivalence relation $\sim$ as follows: $x \sim y$ iff $f(x)=$ $f(y)$ for all continuous function $f: X \rightarrow \mathbb{R}$. By $\phi: X \rightarrow X / \sim$ we denote the mapping which maps $x$ into its equivalence class $[x] \in X / \sim$. Then for each $f \in C(X)$ there exists the unique function $\tilde{f}: X / \sim \rightarrow \mathbb{R}$ such that the following diagram commutes.

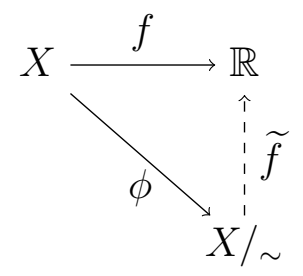

Let $C^{\prime}$ be the family $\{\tilde{f}: f \in C(X)\}$. By endowing $X / \sim$ by the weak topology induced by $C^{\prime}$, GJ76, Theorem 3.7] implies $X / \sim \in T_{3 \frac{1}{2}}$ and that $\phi: X \rightarrow X / \sim$ is continuous. If $X \notin T_{3 \frac{1}{2}}$ is functionally Hausdorff, then $\phi$ is just the identity mapping and the topology on $X / \sim$ is strictly weaker than the topology of $X$.

The mapping $\phi: X \rightarrow X / \sim$ induces the isomorphism $\Phi: C(X / \sim) \rightarrow$ $C(X)$, defined by $f \mapsto f \circ \phi$. By [GJ76, Theorem 1.6] $\Phi$ is also a lattice isomorphism. By applying [GJ76, Theorem 1.9], the restriction of $\Phi$ to $C_{b}(X / \sim)$ induces the isometric isomorphism (which we denote again by $\Phi) \Phi: C_{b}(X / \sim) \rightarrow C_{b}(X)$. 
Remark 6.1. Having in mind that $C_{b}(X)$ is isometric lattice and algebra isomorphic to $C_{b}(X / \sim)$, by Remark 4.1 the classes of $a$-ideals and $o$-ideals coincide in $C_{b}(X)$.

The following lemma explains how ideals of the form $J_{F}^{b}(X)$ are transformed by $\Phi$ and its inverse.

Lemma 6.2. Let $X$ be a Hausdorff space.

(a) For $F \subseteq X$ we have $\Phi^{-1}\left(J_{F}^{b}(X)\right)=J_{\frac{b}{\phi(F)}}(X / \sim)$ and $\Phi^{-1}\left(J_{F}(X)\right)=$ $J_{\overline{\phi(F)}}(X / \sim)$.

(b) For $F \subseteq X / \sim$ we have $\Phi\left(J_{F}^{b}(X / \sim)\right)=J_{\phi^{-1}(F)}^{b}(X)$ and $\Phi\left(J_{F}(X / \sim)\right)=$ $J_{\phi^{-1}(F)}(X)$.

Proof. We provide the proof only for the "bounded" case since the proof for the general case is basically the same.

(a) Take a function $f \in J_{F}^{b}(X)$. Then $\tilde{f} \circ \phi=f$ implies that $\tilde{f} \equiv 0$ on $\phi(F)$. By continuity we conclude $\widetilde{f} \equiv 0$ on $\overline{\phi(F)}$, so that $\Phi^{-1}(f)=$ $\tilde{f} \in J_{\frac{b(F)}{b}}(X / \sim)$. Conversely, if $\tilde{f} \in J_{\overline{\phi(F)}}^{b}(X / \sim)$, then $f=\widetilde{f} \circ \tau \equiv 0$ on $F$.

(b) If $f \in \Phi\left(J_{F}^{b}(X / \sim)\right)$, then $f=\tilde{f} \circ \phi$ for some $\tilde{f} \in J_{F}^{b}(X / \sim)$. Pick $x \in \phi^{-1}(F)$. Then $\phi(x) \in F$ and $f(x)=\tilde{f}(\phi(x))=0$; hence $f \in J_{\phi^{-1}(F)}^{b}(X)$. Conversely, choose $f \in J_{\phi^{-1}(F)}^{b}(X)$. Then $f=\widetilde{f} \circ \phi=$ $\Phi(\widetilde{f})$. Since $\left.f\right|_{\phi^{-1}(F)} \equiv 0$ and $\phi\left(\phi^{-1}(F)\right)=F$ we have $\widetilde{f} \equiv 0$ on $F$, and therefore $f \in \Phi\left(J_{F}^{b}(X / \sim)\right)$.

Theorem 6.3. Let $X$ be a Hausdorff space.

(a) A subspace $J \subseteq C_{b}(X)$ is a projection band iff $J=J_{F}^{b}(X)$ for some clopen set $F \subseteq X$.

(b) A subspace $B \subseteq C(X)$ is a projection band iff $B=J_{F}(X)$ for some clopen subset $F$ in $X$.

Proof. (a) Obviously, $J$ is a projection band in $C_{b}(X)$ iff $\Phi^{-1}(J)$ is a projection band in $C_{b}(X / \sim)$. Therefore, by Theorem $4.4 J$ is a projection band in $C_{b}(X)$ iff there exists a clopen set $F \subseteq X / \sim$ such that $\Phi^{-1}(J)=J_{F}^{b}(X / \sim)$. Therefore, $J$ is a projection band iff it is of the form

$$
J=\Phi\left(J_{F}^{b}(X / \sim)\right)=J_{\phi^{-1}(F)}^{b}(X)
$$


for some clopen set $F$ in $X / \sim$. Since $\phi$ is continuous, $\phi^{-1}(F)$ is clopen. If $J=J_{F}^{b}(X)$ for some clopen subset $F$ of $X$, then by following the lines of the proof of Theorem 4.4(b) we conclude that $J$ is a projection band.

(b) If $B$ is a projection band in $C(X)$, by [Sch74, Proposition 2.2.3], we have $C_{b}(X)=\left(C_{b}(X) \cap B\right) \oplus\left(C_{b}(X) \cap B^{d}\right)$. By [Zaa96, Theorem 11.1], $C_{b}(X) \cap B$ is a projection band in $C_{b}(X)$, so that by Theorem 6.3 there exists a clopen subset $F$ of $X$ with $C_{b}(X) \cap B=J_{F}^{b}(X)$. As in Corollary 4.5 we can prove $B=J_{F}(X)$.

The proof of the converse statement again follows the lines of the proof of Theorem 4.4(b).

The natural question that arises here is whether a closed subset of a Hausdorff space $X$ such that $J_{F}^{b}(X)$ is a band in $C_{b}(X)$ satisfies $F=\overline{\operatorname{Int} F}$. In general, the answer is no.

Example 6.4. Let $\tau_{e}$ be the Euclidean topology on $\mathbb{R}$. Let $X=\mathbb{R}$ be equipped with topology $\tilde{\tau}$ generated by $\mathcal{B}=\tau_{e} \cup\left\{U \backslash N: U \in \tau_{e}\right\}$, where $N=\bigcup_{n=1}^{\infty}\left[\frac{1}{2 n}, \frac{1}{2 n-1}\right]$. The topology $\widetilde{\tau}$ is stronger than $\tau_{e}$ and since $\mathbb{R}$ is a functionally Hausdorff space, $X$ is functionally Hausdorff as well. Therefore, we have $X / \sim=\mathbb{R}$ as sets.

- The topology on $X / \sim$ is stronger than the Euclidean topology.

Indeed, let $F \subseteq \mathbb{R}$ be closed (in the Euclidean topology). Then for every $x \notin F$ there exists a continuous function $\varphi: \mathbb{R} \rightarrow \mathbb{R}$ such that $\varphi(x)=1$ and $\varphi(F)=0$. Since $\varphi: X \rightarrow \mathbb{R}$ is continuous and $x \in\{y \in$ $X:|\varphi(x)-\varphi(y)|<1\} \subseteq X \backslash F, X \backslash F$ is open, and hence $F$ is a closed subset of $X / \sim$.

- The space $X$ is not $T_{3}$.

Indeed, by definition the set $N$ is closed in $X$ and $0 \notin N$. Let $U, V \subseteq X$ be open sets such that $0 \in U$ and $N \subseteq V$. For every $n \in \mathbb{N}, \frac{1}{2 n} \in V$ hence $\left(\frac{1}{2 n}-r_{n}, \frac{1}{2 n}+r_{n}\right) \subseteq V$ for some $r_{n}>0$. So there exists $x_{n} \in$ $\left(\frac{1}{2 n+1}, \frac{1}{2 n}\right) \cap V$. The sequence $\left(x_{n}\right)$ lies in $\mathbb{R} \backslash N$ and inf $x_{n}=0$, hence $\emptyset \neq U \cap\left\{x_{n}: n \in \mathbb{N}\right\} \subset U \cap V$. Therefore we can not separate the point 0 from the closed set $N$.

- $0 \in \mathrm{Cl}_{X / \sim} N=\mathrm{Cl}_{X / \sim}\left(\bigcup_{n=1}^{\infty}\left(\frac{1}{2 n}, \frac{1}{2 n-1}\right)\right)$. 
The equality above should be obvious, so that it remains to prove $0 \in \mathrm{Cl}_{X / \sim} N$. Suppose this is not the case. There exists $f \in C(X)$ such that $f(0)=1$ in $\left.f\right|_{N} \equiv 0$. Then the open sets $f^{-1}\left(\left(\frac{1}{2}, \infty\right)\right)$ and $f^{-1}\left(\left(-\infty, \frac{1}{2}\right)\right)$ form a separation for 0 and $N$ which is not possible from the above.

$$
\text { - } \mathrm{Cl}_{X / \sim} \operatorname{Int}_{X / \sim} F=F \text { where } F=N \cup\{0\} .
$$

Since $F$ is closed in $\mathbb{R}$ and since topologies on $X$ and $X / \sim$ are stronger than the Euclidean topology, $F$ is also closed in both $X$ and $X / \sim$. Therefore we have $\mathrm{Cl}_{X / \sim} \operatorname{Int}_{X / \sim} F \subseteq F$. Because $\mathbb{R} \backslash\{0\}$ and $X \backslash\{0\}$ are homeomorphic they are also homeomorphic to $X / \sim \backslash\{0\}$. Hence $\mathrm{Cl}_{\mathbb{R}} F, \mathrm{Cl}_{X} F$ and $\mathrm{Cl}_{X / \sim} F$ may differ only for the point 0 . The same is true for interiors of the given set. This implies $\operatorname{Int}_{\mathbb{R}} F=\bigcup_{n=1}^{\infty}\left(\frac{1}{2 n}, \frac{1}{2 n-1}\right)$ and so we have $\operatorname{Int}_{X} F=\operatorname{Int}_{X / \sim} F=\bigcup_{n=1}^{\infty}\left(\frac{1}{2 n}, \frac{1}{2 n-1}\right)$. This gives

$$
F \backslash\{0\}=N=\bigcup_{n=1}^{\infty}\left[\frac{1}{2 n}, \frac{1}{2 n-1}\right] \subseteq \mathrm{Cl}_{X / \sim} \operatorname{Int}_{X / \sim} F .
$$

Because $\mathbb{R} \backslash N$ is open in $X$ and $(\mathbb{R} \backslash N) \cap \operatorname{Int}_{X} F=\emptyset$, we have $0 \notin \mathrm{Cl}_{X} \operatorname{Int}_{X} F$, and hence

$$
\mathrm{Cl}_{X} \operatorname{Int}_{X} F=F \backslash\{0\} \subseteq \mathrm{Cl}_{X / \sim} \operatorname{Int}_{X / \sim} F \subseteq \mathrm{Cl}_{\mathbb{R}} \operatorname{Int}_{\mathbb{R}} F=F .
$$

Since $0 \in \mathrm{Cl}_{X / \sim} \operatorname{Int}_{X \sim} F$, we finally conclude $\mathrm{Cl}_{X / \sim} \operatorname{Int}_{X / \sim} F=F$.

- $J_{F}^{b}(X)$ is a band in $C_{b}(X)$; yet $\mathrm{Cl}_{X} \operatorname{Int}_{X} F=F \backslash\{0\}$.

The set $F$ is closed in $X$ and $X / \sim$. The mapping $\phi: X \rightarrow X / \sim$ is the identity mapping as $X$ is functionally Hausdorff. Since the closed ideal $J_{F}^{b}(X)$ satisfies

$$
\Phi^{-1}\left(J_{F}^{b}(X)\right)=J_{\overline{\phi(F)}}^{b}(X / \sim)=J_{F}^{b}(X / \sim)
$$

and since $\mathrm{Cl}_{X / \sim} \operatorname{Int}_{X / \sim} F=F$, we conclude that $J_{F}^{b}(X / \sim)$ is a band in $C_{b}(X / \sim)$. Therefore, $J_{F}^{b}(X)$ is band in $C_{b}(X)$, yet $\mathrm{Cl}_{X} \operatorname{Int}_{X} F=$ $F \backslash\{0\}$.

- $J_{F}^{b}(X)=J_{N}^{b}(X)$ and $N=\overline{\operatorname{Int} N}$.

It is clear that $N=\overline{\operatorname{Int} N}$. Since there is no function $f \in C(X)$ with $\left.f\right|_{N} \equiv 0$ and $f(0) \neq 0$, we conclude $J_{N}^{b}(X) \subseteq J_{F}^{b}(X)$. The opposite inclusion is obvious. 
The topological space constructed in Example 6.4 shows that there are examples of spaces which satisfy weaker separation axioms than $T_{3 \frac{1}{2}}$, and at the same time the conclusion of Proposition 3.3 still holds:

Example 6.5. Let $(X, \widetilde{\tau})$ be the topological space from Example 6.4. We already know that $X$ is functionally Hausdorff but not $T_{3 \frac{1}{2}}$. Take an arbitrary open set $A$ in $X$ and assume $0 \leq f_{\alpha} \nearrow f$ in $C(X)$. We claim that $\left.\left.f_{\alpha}\right|_{A} \nearrow f\right|_{A}$ in $C(A)$. Suppose there is $g: A \rightarrow \mathbb{R}$ such that $\left.f_{\alpha}\right|_{A} \leq g$ and $g<\left.f\right|_{A}$.

Suppose first that there is $0 \neq x \in A$ with $g(x)<f(x)$. Then there exists $r>0$ such that $(x-r, x+r) \subseteq A$ and $g(y)<f(y)$ for each $y \in(x-r, x+r)$. Take any function $\varphi: \mathbb{R} \rightarrow[0,1]$ which is continuous with respect to the Euclidean topology $\tau_{e}$, and satisfies $\varphi(x)=1$ and $\varphi \equiv 0$ on $\mathbb{R} \backslash(x-r, x+r)$. Since $\widetilde{\tau}$ is stronger than $\tau_{e}$, the function $\varphi: X \rightarrow[0,1]$ is also continuous. Then $g \leq\left. f\right|_{A}-\left.\varphi\right|_{A}\left(\left.f\right|_{A}-g\right)$ and $f_{\alpha} \leq f-\varphi(f-g)<f$ for each $\alpha$, where $\varphi(f-g)$ is defined as

$$
\varphi(f-g)(x)=\left\{\begin{array}{cl}
\varphi(x)(f(x)-g(x)) & : x \in A \\
0 & : x \in X \backslash\left[x-\frac{r}{2}, x+\frac{r}{2}\right]
\end{array} .\right.
$$

This is in contradiction with $f_{\alpha} \nearrow f$.

If $0 \in A$ and $g(0)<f(0)$, then $g(y)<f(y)$ on some open neighborhood $U$ of $0 \in X$, so that $g(y)<f(y)$ on some open neighborhood of $y_{0} \in A \backslash\{0\}$ as well. By the previous paragraph this is impossible, and hence $\left.\left.f_{\alpha}\right|_{A} \nearrow f\right|_{A}$.

Question 6.6. Is there an example of a functionally Hausdorff space $X$, an open set $A$ in $X$ and a net $\left(f_{\alpha}\right)$ in $C(X)$ such that $f_{\alpha} \nearrow f$ in $C(X)$ but $\left.\left.f_{\alpha}\right|_{A} \nearrow f\right|_{A}$ does not hold in $C(A)$ ?

If $X \in T_{3 \frac{1}{2}}$, the mapping $F \mapsto J_{F}(X)$ is a one-to-one mapping on the set of all closed subsets of $X$. If $X$ is merely a Hausdorff space, then it is possible that different closed sets induce the same fixed ideal. However, for a set $F \subseteq X$ there is the largest closed set $F^{\prime}$ such that $J_{F}(X)=J_{F^{\prime}}(X)$.

Theorem 6.7. Let $X$ be a Hausdorff space and $F$ a subset of $X$. Then $\phi^{-1}(\overline{\phi(F)})$ is the largest closed set $F^{\prime}$ with the property $J_{F}(X)=$ 
$J_{F^{\prime}}(X)$. Furthermore, we have

$$
J_{F}(X)=J_{\overline{\phi^{-1}(\phi(F))}}(X)=J_{\phi^{-1}(\overline{\phi(F))}}(X) .
$$

Proof. From $J_{\phi(F)}(X / \sim)=J_{\overline{\phi(F)}}(X / \sim)$ and Lemma 6.2 we conclude

$$
J_{\overline{\phi^{-1}(\phi(F))}}(X)=J_{\phi^{-1}(\phi(F))}(X)=J_{\phi^{-1}(\overline{\phi(F))}}(X) .
$$

We claim $J_{F}(X)=J_{\phi^{-1}(\phi(F))}(X)$. Note first that $J_{\phi^{-1}(\phi(F))}(X) \subseteq$ $J_{F}(X)$ follows from the fact $F \subseteq \phi^{-1}(\phi(F))$. For the opposite inclusion, take $f \in J_{F}(X)$ and $x \in \phi^{-1}(\phi(F))$. Then $\phi(x) \in \phi(F)$, so that there is $y \in F$ with $[x]=[y]$. By definition of $\sim$ we have $f(x)=f(y)=0$. This gives $f \equiv 0$ on $\phi^{-1}(\phi(F))$ which proves the claim.

The only thing that remains to be proved is that $\phi^{-1}(\overline{\phi(F)})$ is the largest closed set $G$ with the property $J_{F}(X)=J_{G}(X)$. In order to prove this, let $G$ be an arbitrary closed set with $J_{G}(X)=J_{F}(X)$. Then $J_{\phi(G)}(X / \sim)=J_{\phi(F)}(X / \sim)$. Pick $x \in G$ and assume $\phi(x) \notin \overline{\phi(F)}$. Since $X / \sim \in T_{3 \frac{1}{2}}$, there is $\widetilde{f} \in C_{b}(X / \sim)$ such that $\widetilde{f}(\phi(x))=1$ and $\widetilde{f} \equiv 0$ on $\overline{\phi(F)}$. By Lemma 6.2 we have $f=\Phi_{\phi}(\widetilde{f}) \in J_{\phi^{-1}(\overline{\phi(F))}}(X)=J_{F}(X)=$ $J_{G}(X)$. Since $x \in G$ we have $f(x)=0$ which is a contradiction.

Corollary 6.8. A Hausdorff space $X$ is $T_{3 \frac{1}{2}}$ iff $J_{F}(X)=J_{G}(X)$ for closed subsets $F$ and $G$ implies $F=G$.

Proof. If $X \in T_{3 \frac{1}{2}}$, then the conclusion follows from the second paragraph of Section 3 .

Suppose now that $X \notin T_{3 \frac{1}{2}}$. If $X$ is functionally Hausdorff, then $X / \sim$ and $X$ are equal as sets and $\phi$ is the identity mapping. Since the topology of $X / \sim$ is strictly weaker than the topology of $X, \phi$ is not a closed mapping. Hence, there exists a closed set $F$ in $X$ which is not closed in $X / \sim$. Since $\tau(F)$ is not closed in $X / \sim, F$ is a proper subset of $\phi^{-1}(\overline{\phi(F)})$. By the assumption we have $J_{F}(X) \neq J_{\phi^{-1}(\overline{\phi(F)})}(X)$ which contradicts Theorem 6.7 .

If $X$ is not functionally Hausdorff, there are two different points $x$ and $x^{\prime}$ which cannot be separated by continuous functions. Then $J_{\{x\}}(X)=J_{\left\{x^{\prime}\right\}}(X)$. On the other hand, the assumption on the uniqueness implies $x=x^{\prime}$. Again a contradiction. 
We already mentioned that order complete vector lattices posses the projection property. It is well-known that the converse statement does not hold. Indeed, the set $E$ of all real bounded functions on $[0,1]$ assuming only finitely many different values becomes a vector lattice when ordered pointwise. This vector lattice is not order complete, yet it has the projection property (see e.g. [Zaa96, Exercise 12.6.(ii)]). Order complete vector lattices are also uniformly complete (see e.g. AB03]). Although neither the projection property nor uniform completeness imply order completeness of a given vector lattice, together they do [AB03, Theorem 1.59]. Since by [MN91, Proposition 1.1.8] Banach lattices are uniformly complete, a given Banach lattice $X$ has the projection property iff it is order complete. Moreover, $X$ has order continuous norm iff every closed ideal of $X$ is a band (see e.g. [Zaa96, Theorem 17.17]). In the following result we determine when $C_{b}(X)$ is order continuous. It turns out that whenever $X$ is Hausdorff then $C_{b}(X)$ is order continuous iff $C(X)$ is finite-dimensional and $X$ is pseudocompact; recall that a topological space $X$ is said to be $\boldsymbol{p s e u -}$ docompact whenever every continuous function on $X$ is bounded.

Corollary 6.9. Let $X$ be a Hausdorff space with the property that every closed ideal in $C_{b}(X)$ is a band. Then the following assertions hold.

(a) $X$ is functionally Hausdorff iff $X$ is finite.

(b) $C(X)$ is finite-dimensional and $X$ is pseudocompact.

Proof. (a) By the assumption and Theorem 4.4 every maximal ideal in $C_{b}(X / \sim)$ is of the form $J_{F}^{b}(X / \sim)$ for some closed subset $F$ of $X / \sim$. Hence, $X / \sim$ is compact by Theorem 2.1. Also, for each $x \in X / \sim$ the closed ideal $J_{\{x\}}^{b}(X / \sim)$ is a band, so that $\{x\}$ is open in $X / \sim$, again by Theorem 4.4 . Thus, $X / \sim$ is discrete and since it is also compact, it is finite. Since $X=X / \sim$ as sets, $X$ is finite. The converse statement obviously holds.

(b) By (a) we conclude $X / \sim$ is finite and since $C_{b}(X) \cong C_{b}(X / \sim)$ it follows that $C_{b}(X)$ is finite-dimensional. Let $\left(f_{\lambda}\right)$ be any disjoint system of nonnegative functions in $C(X)$. Then $\left(f_{\lambda} \wedge \mathbb{1}\right)$ is a disjoint system in $C_{b}(X)$ and since $C_{b}(X)$ is finite-dimensional, $\left(f_{\lambda} \wedge \mathbb{1}\right)$ is finite. Since $\mathbb{1}$ is a weak unit in $C(X)$, we conclude $\left(f_{\lambda}\right)$ is finite, so that $C(X)$ is 
finite-dimensional by [LZ71, Theorem 26.10]. Hence, $C_{b}(X)$ is a finitedimensional ideal in $C(X)$. Since $C_{b}(X)$ is also order dense in $C(X)$, we conclude $C_{b}(X)=C(X)$.

\section{LIFTING UN-CONVERGENCE}

In this section we apply our results to the so-called problem of "lifting un-convergence". First we recall some basic facts needed throughout this section.

If $E$ is a Banach lattice, then a net $\left(x_{\alpha}\right)$ is said to un-converge to a vector $x \in E$ whenever for each $y \in E_{+}$we have $\left|x_{\alpha}-x\right| \wedge y \rightarrow 0$ in norm. We write $x_{\alpha} \stackrel{\text { un }}{\longrightarrow} x$ whenever the net $\left(x_{\alpha}\right)$ un-converges to $x$. This mode of convergence was introduced by V.G. Troitsky [Tro04] under the name of $d$-convergence. In [Tro04, Example 20] it was proved that un-convergence in $C_{0}(X)$ coincides with the uniform convergence on compacta of $X$ whenever $X$ is normal. In particular, when $X$ is a compact Hausdorff space, then un-convergence coincides with the uniform convergence. In the case of $L_{p}(\mu)$-spaces with $\mu$ finite he proved that un-convergence coincides with convergence in measure [Tro04, Example 23]. It is a standard fact from measure theory that a sequence $\left(f_{n}\right)$ converging in measure to $f$ always has a subsequence converging to $f$ almost everywhere (see e.g. [Fol99, Theorem 2.30]). In [GTX17, Proposition 3.1] the authors proved that a sequence $\left(f_{n}\right)$ in $L_{0}(\mu)$ converges almost everywhere to $f \in L_{0}(\mu)$ whenever $\left|f_{n}-f\right| \wedge g \rightarrow 0$ in order of $L_{0}(\mu)$ for each nonnegative $g \in L_{0}(\mu)$. The latter mode of convergence goes back to [Nak48, DeM64, Kap97]. Kaplan referred to this convergence as unbounded order convergence or uo-convergence for short. Until very recently unbounded order convergence was not studied actively and was left out from the active area of research. The systematic study of this mode of convergence and its properties started with papers of Gao, Troitsky and Xanthos [Gao14, GX14, GTX17] and others. The systematic study and properties of un-convergence started in [DOT]. Among other things, authors proved that un-convergence is topological. On the other hand, uo-convergence is not (see e.g. Ord66]). In [KMT17] the authors initiated the study of un-topology, 
i.e., the topology given by un-convergence. They posed the following question.

Question 7.1. Let $B$ be a band in a Banach lattice $E$. Suppose that every net in $B$ which is un-null in $B$ is also un-null in $E$. Does this imply that $B$ is a projection band?

When the norm of $E$ is order continuous, every band is a projection band. It was proved in [KMT17] that in this case a net $\left(x_{\alpha}\right)$, which is un-null in a sublattice of $E$ it is also un-null in $E$. [KMT17, Example 4.2] shows that there exists a band $B$ in $C[-1,1]$ and a un-null net $\left(x_{\alpha}\right)$ in $B$ which is not un-null in $C[-1,1]$.

The following theorem provides a positive answer to Question 7.1 for a Banach lattice $C_{b}(X)$ of all bounded continuous functions on a Hausdorff space.

Theorem 7.2. Let $X$ be a Hausdorff space and $J$ a closed ideal in $C_{b}(X)$. Suppose that every un-null net in $J$ is also un-null in $C_{b}(X)$. Then $J$ is a projection band in $C_{b}(X)$.

Proof. We first consider the case when $X$ is compact. Since $J$ is a closed ideal in $C(X)$, by Theorem 2.2 there exists a closed set $F$ such that $J=J_{F}(X)$. If $F$ is not open, there exists $x \in F \backslash \operatorname{Int} F$. Let $\mathcal{B}_{x}=\left\{W_{\lambda}\right\}_{\lambda \in \Lambda}$ be the set of all open neighborhoods of the point $x$. Since $X$ is Hausdorff, Dug66, VII.I.2] implies

$$
\{x\}=\bigcap_{\lambda \in \Lambda} W_{\lambda} .
$$

If $\Lambda$ is finite, $\{x\}$ is open, so that $x \in \operatorname{Int} F$ which is a contradiction. Therefore $\Lambda$ is infinite.

Since $x \in F \backslash \operatorname{Int} F$, for each $\lambda \in \Lambda$ the set $W_{\lambda} \backslash F$ is nonempty. Pick any $x_{\lambda} \in W_{\lambda} \backslash F$. Also, let $V_{\lambda}$ be an arbitrary open neighborhood of $x_{\lambda}$ in $(X \backslash F) \cap W_{\lambda}$. By Urysohn's lemma, for each $\lambda \in \Lambda$ there exists a nonnegative continuous function $f_{\lambda}$ such that $f_{\lambda}\left(x_{\lambda}\right)=1$ and $\left.f\right|_{X \backslash V_{\lambda}} \equiv 0$. We define an ordering on the set $\Lambda$ by $\lambda \leq \mu$ iff $W_{\mu} \leq W_{\lambda}$. With this ordering $\Lambda$ becomes a directed set.

We claim that $f_{\lambda} \stackrel{\text { un }}{\longrightarrow} 0$ in $J$. Pick an arbitrary function $g \in J$. Then $g(x)=0$. Continuity of $g$ implies that for every $\epsilon>0$ there exists an 
open neighborhood $U$ of $x$ such that $|g(y)|<\epsilon$ for each $y \in U$. There exists $\lambda_{0} \in \Lambda$ such that $W_{\lambda_{0}} \subseteq U$. If $\lambda \geq \lambda_{0}$, then $V_{\lambda} \subseteq W_{\lambda} \subseteq W_{\lambda_{0}} \subseteq U$. If $y \in X \backslash V_{\lambda}$, then $f_{\lambda}(y)=0$ and if $y \in V_{\lambda}$, then $|g(y)|<\epsilon$. We conclude $f_{\lambda} \wedge|g|<\epsilon$ for all $\lambda \geq \lambda_{0}$. Therefore, $f_{\lambda} \stackrel{\text { un }}{\longrightarrow} 0$ in $J$.

Now we claim that the net $\left(x_{\lambda}\right)$ converges to $x$. Indeed, let $W$ be an arbitrary neighborhood of $x$. Then there exists $\lambda_{0}$ such that $W_{\lambda_{0}} \subseteq W$. If $\lambda \geq \lambda_{0}$, then $x_{\lambda} \in W_{\lambda} \subseteq W_{\lambda_{0}} \subseteq W$ which proves the claim.

The assumption $f_{\lambda} \stackrel{\text { un }}{\rightarrow} 0$ in $J$ implies that $f_{\lambda} \rightarrow 0$ in $C(X)$ which contradicts the fact that $f_{\lambda}\left(x_{\lambda}\right)=1$ for each $\lambda \in \Lambda$. Therefore $F=$ Int $F$ is clopen; hence $J$ is a projection band by Theorem 2.3 .

The general case follows from the first part of the proof and that whenever $X$ is "just" Hausdorff, then $C_{b}(X)$ and $C(\beta(X / \sim))$ are isometrically algebra and lattice isomorphic.

The following corollary follows immediately from Theorem 7.2 and Theorem 6.3.

Corollary 7.3. Let $X$ be a Hausdorff space and $x \in X$ such that every un-null net in $J_{\{x\}}^{b}(X)$ is also un-null in $C_{b}(X)$. Then $x$ is isolated in $X$.

We proceed with an application of Corollary 6.9 and Theorem 7.2 .

Corollary 7.4. Let $X$ be a Hausdorff space with the property that for every maximal ideal $J$ in $C_{b}(X)$, every un-null net in $J$ is un-null in $C_{b}(X)$. Then $X$ is finite.

Proof. Since every point in $X$ is isolated, by Corollary $7.3, X$ is discrete and therefore $X \in T_{3 \frac{1}{2}}$. Let $J$ be a closed ideal in $C(\beta X)$. Then $\beta^{-1}(J)$ is a closed ideal in $C_{b}(X)$. Since every un-null net in $\beta^{-1}(J)$ is un-null in $C_{b}(X)$, every un-null net in $J$ is also un-null in $C(\beta X)$. Theorem 7.2 implies $J$ is a projection band in $C(\beta X)$. Corollary 6.9 implies $\beta X$ is finite and so $X$ is finite.

We conclude this paper with $C_{0}(X)$-analogs of results of this section. Since the proofs are basically the same as in the $C_{b}(X)$-case, we omit them.

Theorem 7.5. Let $X$ be a locally compact Hausdorff space. 
(a) Let $J$ be a closed ideal in $C_{0}(X)$. If every un-null net in $J$ is also un-null in $C_{0}(X)$, then $J$ is a projection band in $C_{0}(X)$.

(b) For $x \in X$, if every un-null net in $J_{\{x\}}^{0}(X)$ is un-null in $C_{0}(X)$, then $x$ is isolated in $X$.

(c) If for every maximal ideal $J$ in $C_{0}(X)$ every un-null net in $J$ is un-null in $C_{0}(X)$, then $X$ is discrete.

Acknowledgments: The authors would like to thank the reviewers for many valuable comments which helped to improve the quality of the paper in the present form.

\section{REFERENCES}

[AB03] C.D. Aliprantis and O. Burkinshaw, Locally solid Riesz spaces with applications to economics, 2nd ed., AMS, Providence, RI, 2003.

[AB06] C.D. Aliprantis and O. Burkinshaw, Positive operators, 2nd edition, Springer 2006.

[ABo06] C.D. Aliprantis and K.C. Border, Infinite dimensional analysis. A hitchhiker's guide, 3th ed., Springer, Berlin, 2006. xxii+703 pp.

[AP74] A. V. Arkhangel'skii, V. I. Ponomarev, Fundamentals of general topology in problems and exercises, Izdat. "Nauka", Moskva, 1974,(in Russian)(English translation: ser. Mathematics and its Applications, D. Reidel Publishing Co., Dordrecht-Boston, Mass., 1984. )

[Con99] J.B. Conway, A course in functional analysis, 2nd edition, SpringerVerlag, New York, 1990.

[DeM64] R. DeMarr, Partially ordered linear spaces and locally convex linear topological spaces, Illinois J. Math. 8, 1964, 601-606.

[DOT] Y. Deng, M. O'Brien, and V.G. Troitsky, Unbounded norm convergence in Banach lattices, Positivity 21 (2017), 963-974.

[Dug66] J. Dugundji, Topology, Allyn and Bacon, Inc., Boston, Mass. 1966.

[Fol99] G.B. Folland, Real analysis: Modern techniques and their applications, 2nd edition, Pure and Applied Mathematics, John Wiley \& Sons, Inc., New York, 1999.

[Gao14] N. Gao, Unbounded order convergence in dual spaces, J. Math. Anal. Appl., 419 (2014), 347-354.

[GJ76] L. Gillman, M. Jerison, Rings of Continuous Functions, Graduate Texts in Math. 43, Springer-Verlag, Berlin-Heidelberg-New York, 1976.

[GX14] N. Gao and F. Xanthos, Unbounded order convergence and application to martingales without probability, J. Math. Anal. Appl. 415 (2014), 931-947. 
[GTX17] N. Gao, V.G. Troitsky, and F. Xanthos, Uo-convergence and its applications to Cesàro means in Banach lattices, Israel J. Math., 220 (2017), 649-689.

[HS12] N. Hindman and D. Strauss, Algebra in the Stone-Čech compactification. Theory and applications, de Gruyter, 1998, Second revised and extended edition 2012.

[KMT17] M. Kandić, M.A.A. Marabeh, V.G.Troitsky, Unbounded norm topology in Banach lattices, J. Math. Anal. Appl. 451 (2017), no. 1, 259-279.

[Kan09] E. Kaniuth, A course in commutative Banach algebras, Graduate Texts in Math. 246, Springer, New York, 2009.

[Kap97] S. Kaplan, On Unbounded Order Convergence, Real Anal. Exchange 23(1), 1997, 175-184.

[LZ71] W.A.J. Luxemburg, A.C. Zaanen, Riesz spaces I, North-Holland Mathematical Library, 1971.

[MN91] P. Meyer-Nieberg, Banach lattices, Springer-Verlag, Berlin, 1991.

[Nak48] H. Nakano, Ergodic theorems in semi-ordered linear spaces, Ann. of Math. (2), 49, 1948, 538-556.

[Ord66] E. T. Ordman, Convergence almost everywhere is not topological, American Math. Monthly 73 (1966), no. 2, 1882-183.

[Sch74] H.H. Schaefer, Banach Lattices and Positive Operators, Die Grundlehren der mathematischen Wissenschaften, vol. 215, SpringerVerlag, New York, 1974.

[Tro04] V. G. Troitsky, Measures on non-compactness of operators on Banach lattices, Positivity 8 (2004), no. 2, 165-178.

[Zaa96] A.C. Zaanen, Introduction to Operator Theory in Riesz Spaces, Springer, Berlin - Heidelberg - New York (1996).

Fakulteta Za Matematiko in Fiziko, Univerza v LuUbljani, Jadranska ulica 19, SI-1000 Ljubluana, Slovenija

E-mail address: marko.kandic@fmf.uni-lj.si

Fakulteta za Matematiko in Fiziko, Univerza v Ljubljani, Jadranska Ulica 19, SI-1000 Ljubluana, Slovenija

E-mail address: ales.vavpetic@fmf.uni-lj.si 